\title{
Design and Analysis of the GCRA Traffic Shaper for VBR Services in ATM Networks*
}

\author{
Mingfu Li Zsehong Tsai ${ }^{\dagger}$ \\ Department of Electrical Engineering, Rm. 543 \\ National Taiwan University, Taipei, Taiwan, R.O.C. \\ E-mail: lmf@eagle.ee.ntu.edu.tw, ztsai@cc.ee.ntu.edu.tw
}

Tel: 886-2-3635251, 886-2-3625252 Ext. 543

Fax: 886-2-3638247

\begin{abstract}
The proposed GCRA traffic shaper consists of a regulator and a scheduler. It can shape multiple incoming VBR cell streams simultaneously to be strictly conforming according to the GCRA algorithm when the cells depart for the ATM output link. The impact of cell emission conflicts is considered and resolved by using a feedback signal from the scheduler to the regulator. The call admission control condition and the cell delay bound are derived. Simulation results demonstrate that the output cell streams of the proposed GCRA traffic shaper do not contain any non-conforming cells and the output queue size is significantly reduced.
\end{abstract}

\section{Introduction}

Since the traffic management of ATM networks became an important research area, many traffic shapers or cell spacing devices have been proposed to reduce the burstiness of the VBR traffic $[1,2,3,4,5]$. The concept of traffic shaper or cell spacer is to store the input cells in the buffer and then output them at eligible time or theoretical reemission time. Traffic shapers can be placed in the source node, in the network entry point or any intermediate multiplexing nodes, for example, the ATM switches. In $[1,2]$, traffic shapers or cell spacers are designed according to the socalled Virtual-Scheduling Algorithm (VSA) to reduce the cell delay variation (CDV) due to queueing in multiplexing nodes. In [3], Brochin employs a cell spacing device to prevent any two successive cells from being transmitted within a time shorter than a variable value. Traffic shaper is implemented by the sliding window mechanism followed by a peak bit rate reduction mechanism in [4]. In [5], two level shaping algorithm which depends on the buffer occupancy of the shaper is proposed.

*This work was supported by National Science Council of the Republic of China under Grant NSC 86-2215-E-002-033.

tCorresponding Author
In most of these papers, the assumed models include multiple traffic streams passing through separate cell spacing devices and a second-stage multiplexer before output. Since the impact of the multiplexer on the output traffic usually is not taken into account, the output cell streams may be non-conforming due to emission conflicts within the multiplexer, regardless the fact that the cell streams observed at the output of spacing devices are conforming. In other words, such emission conflicts are likely to lead to the unnecessary peak or sustainable cell rate violation when cells are transmitted to the ATM output link. It reveals that the multiplexer should be modeled as a component of the traffic shaper such that the impact of cell output conflict can be resolved. In [6], a software method is proposed for multiplexing spacer outputs on cell emissions. Unfortunately, the output cell streams of the multiplexer are still not guaranteed to be conforming. Thus, the design of traffic shapers which can regulate the peak cell rate, sustainable cell rate and burst tolerance simultaneously to be conforming is still at issue.

In this paper, we proposed the GCRA traffic shaper which can shape multiple incoming VBR traffic streams simultaneously to be strictly conforming with respect to the GCRA algorithm when the cells enter the ATM output link. Since the shaping delay is related to both the input and output traffic descriptors, we will first derive the call admission control condition to guarantee the shaper performance and then analyze the total shaping delay bound later.

The organization of this paper is as follows. In section 2 , the traffic model is defined. In section 3 , we describe the design of the GCRA traffic shaper. In section 4 , the delay bound of the GCRA traffic shaper is derived. In section 5 , the proposed GCRA traffic shaper is demonstrated by numerical examples. And finally in section 6, we present our conclusions. 


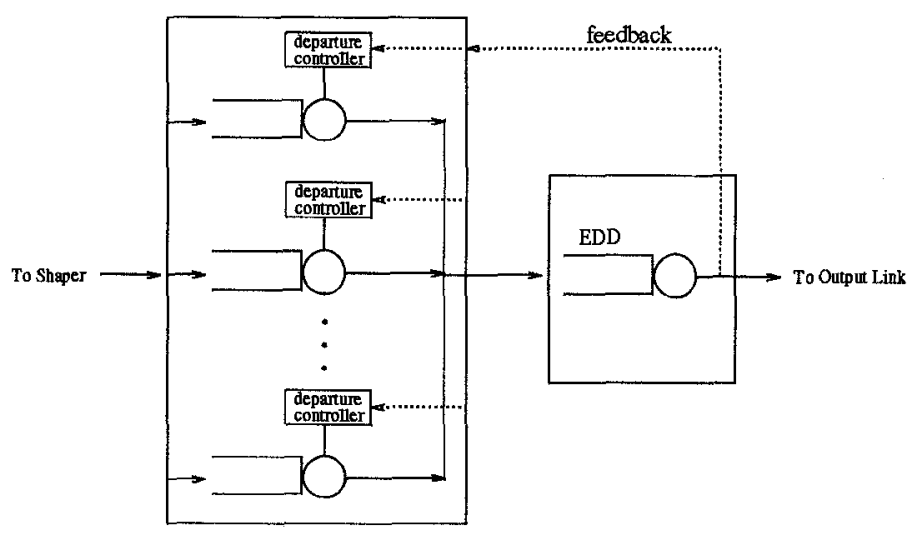

regulator

scheduler

Figure 1: The model of the GCRA traffic shaper.

\section{The Traffic Model}

We use the concept of traffic specification in the ATM Forum UNI Specification [7] to define the traffic descriptor $(X, T, \tau)$ for each virtual connection (VC). Here, $X$ indicates the minimum cell interarrival time, which corresponds to the peak cell rate parameter, and $T$ represents the minimum average cell interarrival time. Note that the inverse of $T$ is equivalent to the sustainable cell rate. The burst tolerance $\tau$ is the maximal amount of time that is allowed for the actual cell arrival to be earlier than the cell arrival time theoretically predicated, under the assumption that any two consecutive cells are separated by the interval $T$. The latter burst tolerance parameter $\tau$ is identical to that defined in Forum's GCRA definition [7]. In other words, the traffic descriptor $(X, T, \tau)$ indicates the following traffic characteristics of a cell stream: regarding the sustainable cell rate, the cell stream conforms to GCRA $(T, \tau)$; when the peak cell rate is concerned, the cell interarrival time is strictly larger or equal to $X$, i.e., conforms to $\operatorname{GCRA}(X, 0)$. In the rest of this paper, the desired output traffic descriptor of VC $j$ is specifically specified as $\left(X_{j}, T_{j}, \tau_{j}\right)$.

\section{The GCRA Traffic Shaper}

\subsection{GCRA Traffic Shaper Model}

In this section, we propose a new traffic shaper model which consists of two components: a regulator and a scheduler, as shown in Fig. 1. The regulator consists of several departure controllers. Each departure controller regulates the peak cell rate, sustainable cell rate and burst tolerance for one $\mathrm{VC}$ according to its individual output traffic descriptor. The operation of the traffic shaper follows a discrete timeaxis, on which the normalized cell emission time is used as a time slot. If a cell arrives early, the regulator will stop it in the buffer. The latter cell then enters the scheduler at its eligible time. The scheduler is responsible for resolving the emission conflicts between cells from different $\mathrm{VCs}$ and transmits cells to the network. When a cell reaches the HOL position in the scheduler, it can be transmitted at the beginning of the next slot. Hence, the minimum sojourn time at the scheduler for any cell is one slot. In order to prevent the cell emissions from violating the peak cell rate constrain$t$, there exists a feedback signal from the scheduler to the GCRA mechanism within the regulator. This assures that the calculation of the eligible time of the next cell emission of the same VC is always done after the receipt of the feedback signal triggered by the current emission. Therefore, there can be at most one cell in the scheduler for each VC at any time. The buffer size in the scheduler is thus determined by the maximal number of VCs that the GCRA traffic shaper is designed to support.

\subsection{Algorithm of the GCRA Traffic Shaper}

In order to describe the GCRA shaping algorithm in the regulator in details, some control variables for shaped VCs are defined as follows. The $k$-th cell from VC $j$ arrives at the shaper (or regulator) at time $A_{j}(k)$ and is buffered until the Eligible Time $E T_{j}(k)$ before it enters the scheduler. It is then attached an Initial Due-Date $I D D_{j}(k)$ when it enters the scheduler. Then it is targeted to leave the scheduler at the Theoretical Departure Time $T D T_{j}(k)$. However, the time epoch it leaves the scheduler could be different. We denote the Actual Departure Time for the $k$-th cell of VC $j$ as $A D T_{j}(k)$. During the operation of the traffic shaper, these variables are calculated by the following iterative form

$$
\begin{array}{r}
T D T_{j}(k)=\max \left\{A D T_{j}(k-1), T D T_{j}(k-1)\right\}+T_{j}, \quad(1) \\
E T_{j}(k)=\max \left\{A D T_{j}(k-1)+X_{j}-1, T D T_{j}(k)-\tau_{j}-1, A_{j}(k)\right\} \\
I D D_{j}(k)=\max \left\{T D T_{j}(k)-E T_{j}(k), \delta_{j}\right\},
\end{array}
$$

with initial values $T D T_{j}(1)=A_{j}(1), E T_{j}(1)=A_{j}(1)$, $I D D_{j}(1)=\delta_{j}$, where $\delta_{j}$ is the minimal initial due-date, given by $\delta_{j}=\min \left\{T_{j}-X_{j}, \tau_{j}\right\}+1$.

First consider only a specific VC, say VC $j$. When the $k$-th cell arrives at the regulator, $T D T_{j}(k)$ is not determined until the feedback signal triggered by the departure of the $(k-1)$-th cell is received. $T D T_{j}(k)$ is then set to $\max \left\{A D T_{j}(k-1), T D T_{j}(k-1)\right\}+T_{j}$ so that it meets the output sustainable cell rate constraint of VC $j$. In order to guarantee the output cell stream to conform to $\left(X_{j}, T_{j}, \tau_{j}\right)$, the $k$-th cell can not enter the scheduler earlier than either the time epoch $A D T_{j}(k-1)+X_{j}-1$ or $T D T_{j}(k)-\tau_{j}-1$; otherwise, it might violate the peak cell rate constraint or the burst tolerance constraint, respectively. By taking account of the fact that a cell can never enter the scheduler before it arrives at the regulator, Eligible Time can be expressed as equation (2). Actually, equation (2) implies that a cell is allowed to enter the scheduler as early as possible. Thus, such a traffic shaper implementation strategy can be called the Most Greedy Method (MGM). Under ideal operation conditions, the maximal throughput of all VCs can be maintained with the use of the traffic shaper. This requires that no cell losses occur in scheduler and all cells to be emitted before their Theoretical Departure Times are reached. The first requirement can be easily met by allocating $M$ buffers at the scheduler, where $M$ is the maximal number of VCs that the shaper supports. To satisfy the second requirement, the 


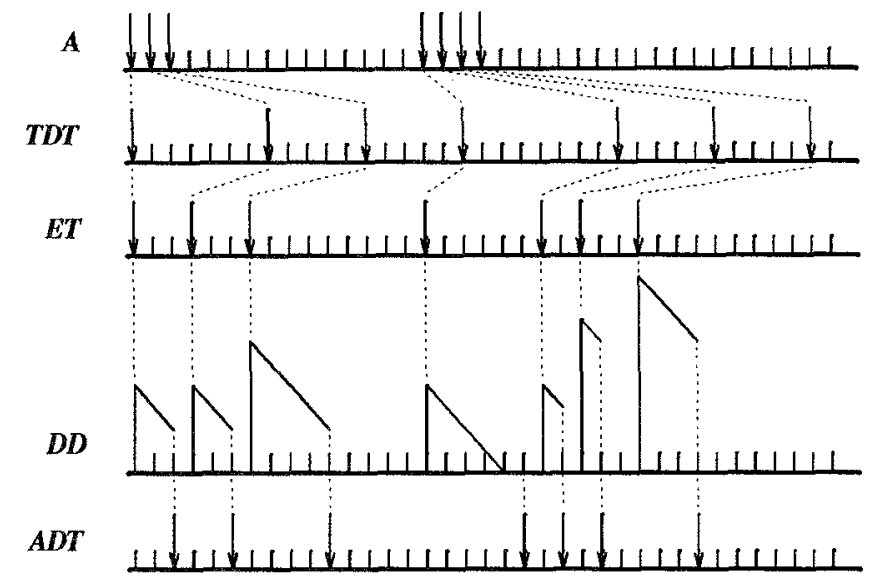

Figure 2: Sample-path of a specific VC with traffic descriptor $(X, T, \tau)=(2,5,8)$.

difference between $T D T_{j}(k)$ and $E T_{j}(k)$ is used as the preliminary initial due-date whenever the shaper is nonempty with respect to VC $j$. From (1) and (2), it can be shown that $T D T_{j}(k)-E T_{j}(k)$ can not be less than $\delta_{j}$ unless the $k$-th cell of VC $j$ observed no cells of VC $j$ in the scheduler and regulator. The proof is left to the readers. The final initial duedate, as given by equation (3), is set to be the maximum of preliminary initial due-date $T D T_{j}(k)-E T_{j}(k)$ and the minimal initial due-date $\delta_{j}$. The latter is used to reduce overdue condition in case of output conflicts. From (1) to (3), it can be shown that $I D D_{j}(k)$ satisfies $\tau_{j}+1 \geq I D D_{j}(k) \geq \delta_{j}$. After the $k$-th cell enters the buffer of the scheduler, its instantaneous due-date at time $t$, denoted as $D D_{j}(k, t)$, is defined to be the difference between $I D D_{j}(k)$ and the waiting time $t-E T_{j}(k)$ in the scheduler. Hence, it satisfies

$$
D D_{j}(k, t)=I D D_{j}(k)-\left[t-E T_{j}(k)\right], \text { for } t \geq E T_{j}(k) .
$$

If $D D_{j}(k, t)=0$, it maintains the value zero from $t$ on. We say that a cell is overdue if its due-date is zero and still resides at the scheduler.

In the scheduler, the Earliest-Due-Date (EDD) service discipline [8] is employed to minimize the number of overdue events. The cell with the smallest due-date is emitted first. If multiple cells have equal due-date, they are emitted in a FIFO order. Fig. 2 shows one sample-path of the control variables associated with a specific VC.

\section{Performance Analysis}

\subsection{Delay Bound in the Scheduler}

Theorem 1 : Assume that the number of active VCs is g. The output traffic descriptor of $V C j$ is $\left(X_{j}, T_{j}, \tau_{j}\right), j=$ $1, \cdots, g$. Consider a target cell from $V C j$ with initial duedate $y$. Then the delay bound, $d_{j}(y)$, of the target cell in the scheduler is given as follows.

$$
d_{j}(y)=\max _{\Delta \geq 0}\left\{N_{j}(\Delta)+\sum_{i=1, i \neq j}^{g} N_{i}\left(y+\Delta-\delta_{i}\right)-\Delta\right\},
$$

where $N_{i}(t)=\min \left\{\left\lfloor 1+\frac{t}{X_{i}}\right\rfloor,\left\lfloor 1+\frac{t+\tau_{i}}{T_{i}}\right\rfloor\right\}$, for $t \geq 0 ; N_{i}(t)=$ 0 , otherwise.

Proof : Consider the target cell from VC $j$ which enters the scheduler with initial due-date $y$ at time $t$. Assume the busy period that contains time $t$ started at earlier time $(t-\Delta)$. Then the last cell from VC $i(i \neq j)$ that can delay the target cell must have arrived before time $(t+y-s)$, where $s\left(s \geq \delta_{i}\right)$ is the initial due-date of this cell. Hence, between time $(t-\Delta)$ and time $(t+y-s)$ the maximum number of cells that do not belong to VC $j$ and will be emitted before the target cell is equal to

$$
\sum_{i=1, i \neq j}^{g} N_{i}(y+\Delta-s) \leq \sum_{i=1, i \neq j}^{g} N_{i}\left(y+\Delta-\delta_{i}\right) .
$$

Moreover, the number of cells which belong to VC $j$ and enter the scheduler during $[t-\Delta, t]$ is bounded by $N_{j}(\Delta)$. Since both the VC $i$ (for all $i \neq j$ ) cells entering the scheduler during $[t-\Delta, t+y-s]$ and the VC $j$ cells entering the scheduler during $[t-\Delta, t]$ contribute to the delay of the target cell, we have the delay bound equation (5).

\subsection{Call Admission Control}

The following theorem provides the sufficient condition to avoid cell overdue. One should note that if a cell is overdue, then its $A D T$ is larger than its $T D T$. However, $A D T>T D T$ doesn't always imply an overdue event.

Theorem 2: If $d_{j}\left(\delta_{j}\right) \leq \delta_{j}$, then no cell of $V C j$ will be overdue.

Proof : Assume that the number of active VCs is $g$. Consider an arbitrary cell from VC $j$ and let its initial due-date be $y$. If $d_{j}(y) \leq y$, for all $y \geq \delta_{j}$, this cell shall not experience overdue events. To show the sufficient condition, by equation (5) one can express $d_{j}(y)-y$ as

$$
\begin{aligned}
& d_{j}(y)-y \\
& =\max _{\Delta \geq 0}\left\{N_{j}(\Delta)+\sum_{i=1, i \neq j}^{g} N_{i}\left(y+\Delta-\delta_{i}\right)-(y+\Delta)\right\} \\
& \leq \max _{\Delta \geq y-\delta_{j}}\left\{N_{j}(\Delta)+\sum_{i=1, i \neq j}^{g} N_{i}\left(\delta_{j}+\Delta-\delta_{i}\right)-\left(\delta_{j}+\Delta\right)\right\} .
\end{aligned}
$$

For $y \geq \delta_{j}$, one can write

$$
\begin{aligned}
& d_{j}(y)-y \\
& \leq \max _{\Delta \geq 0}\left\{N_{j}(\Delta)+\sum_{i=1, i \neq j}^{g} N_{i}\left(\delta_{j}+\Delta-\delta_{i}\right)-\left(\delta_{j}+\Delta\right)\right\} \\
& =d_{j}\left(\delta_{j}\right)-\delta_{j} .
\end{aligned}
$$

Thus, $d_{j}(y) \leq y$ for all $y \geq \delta_{j}$ is implied by $d_{j}\left(\delta_{j}\right) \leq \delta_{j}$. We then conclude $d_{j}\left(\delta_{j}\right) \leq \delta_{j}$ implies no cell overdue for VC $j$.

The call admission control procedure operates as follows. When a new VC, say VC $n$, requests to be supported by the shaper, $d_{j}\left(\delta_{j}\right)$ is computed for all existing VCs and VC $n$. If all $d_{j}\left(\delta_{j}\right) \leq \delta_{j}$ are satisfied, the new request is granted. Otherwise, it is rejected. 


\subsection{Delay Bound of the GCRA Traffic Shaper}

Lemma 1 : If no cell from $V C j$ is overdue, then $A D T_{j}(k)>T D T_{j}(k)$ implies $I D D_{j}(k)=\delta_{j}$.

Proof : Since no cell from VC $j$ is overdue, the cell departure must be early enough to satisfy

$$
A D T_{j}(k) \leq E T_{j}(k)+I D D_{j}(k) .
$$

Assume that $I D D_{j}(k)>\delta_{j}$, then from Eq. (3) one can obtain

$$
T D T_{j}(k)=E T_{j}(k)+I D D_{j}(k) \geq A D T_{j}(k),
$$

which is a conflict with $A D T_{j}(k)>T D T_{j}(k)$. Hence, $I D D_{j}(k)=\delta_{j}$ must hold.

Theorem 3 : Assume the output and input traffic descriptors of $V C j$ are given by $\left(X_{j}, T_{j}, \tau_{j}\right)$ and $\left(X_{j}^{\prime}, T_{j}^{\prime}, \tau_{j}^{\prime}\right)$, where $T_{j} \leq T_{j}^{\prime}$. If no overdue for $V C j$ is guaranteed, the cell delay of $V C j$ within the whole GCRA traffic shaper is bounded by $D_{j}$ which satisfies

$$
D_{j} \leq \delta_{j}+\left\lfloor\frac{T_{j}^{\prime}}{T_{j}^{\prime}-X_{j}^{\prime}}\right\rfloor\left(T_{j}-X_{j}^{\prime}\right) .
$$

If $T_{j}=T_{j}^{\prime}$, then

$$
D_{j} \leq \delta_{j}+\tau_{j}^{\prime} .
$$

Proof : Consider an arbitrary interval $\left[A_{j}(m+1), A_{j}(m+\right.$ $L)$ ), where $m$ and $L(L \geq 2)$ are integers, satisfying $A D T_{j}(m+1)>T D T_{j}(m+1), A D T_{j}(m+n) \leq T D T_{j}(m+n)$ for $2 \leq n<L$ and $A D T_{j}(m+L)>T D T_{j}(m+L)$, as shown in Fig. 3. Notice that a sequence of such intervals should have represented all possible cell arrival and departure patterns. From (1), it is obvious that $T D T_{j}(m+2)=$ $A D T_{j}(m+1)+T_{j}$ and $T D T_{j}(m+n)=T D T_{j}(m+n-1)+T_{j}$ for $3 \leq n<L$. Since $A D T_{j}(m+1)>T D T_{j}(m+1)$, from Lemma $1 I D D_{j}(m+1)=\delta_{j}$ and one can show that $E T_{j}(m+1)=A_{j}(m+1)$. Thus, the $(m+1)$-th cell does not experience any delay in the regulator. If no cells from VC $j$ are overdue, we conclude that the delay of the $(m+1)$-th cell can not be larger than $\delta_{j}$. Hence, for $2 \leq n<L$, we have

$$
\begin{aligned}
T D T_{j}(m+n) & =A D T_{j}(m+1)+(n-1) T_{j} \\
& \leq A_{j}(m+1)+\delta_{j}+(n-1) T_{j} .
\end{aligned}
$$

On the other hand, with input traffic descriptor $\left(X_{j}^{\prime}, T_{j}^{\prime}, \tau_{j}^{\prime}\right)$, it can be shown that

$$
A_{j}(m+n) \geq \begin{cases}A_{j}(m+1)+(n-1) X_{j}^{\prime}, & n \leq M B S_{j}^{\prime} \\ A_{j}(m+1)+(n-1) T_{j}^{\prime}-\tau_{j}^{\prime}, & n>M B S_{j}^{\prime}\end{cases}
$$

where $M B S_{j}^{\prime}$ is the maximum burst size of the $j$-th input $\mathrm{VC}[7]$ and is equal to

$$
M B S_{j}^{\prime}=\left\lfloor 1+\frac{\tau_{j}^{\prime}}{T_{j}^{\prime}-X_{j}^{\prime}}\right\rfloor .
$$
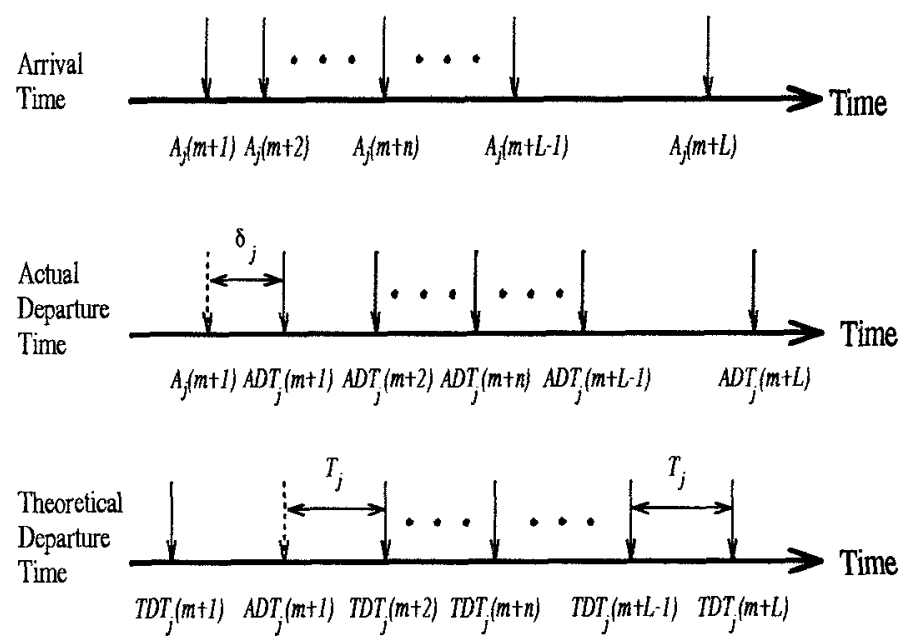

Figure 3: A time diagram for $A_{j}(k), A D T_{j}(k)$ and $T D T_{j}(k)$.

By definition, $D_{j}$ satisfies

$$
\begin{aligned}
D_{j}= & \max _{L>n \geq 1}\left\{A D T_{j}(m+n)-A_{j}(m+n)\right\} \\
\leq & \max \left\{A D T_{j}(m+1)-A_{j}(m+1),\right. \\
& \left.\max _{L>n \geq 2}\left\{T D T_{j}(m+n)-A_{j}(m+n)\right\}\right\} .
\end{aligned}
$$

By combining (11), (12) and (14), the delay bound for arbitrary $L$ becomes

$$
D_{j} \leq \max _{n \geq 1} \begin{cases}\delta_{j}+(n-1)\left(T_{j}-X_{j}^{\prime}\right), & n \leq M B S_{j}^{\prime} \\ \delta_{j}+(n-1)\left(T_{j}-T_{j}^{\prime}\right)+\tau_{j}^{\prime}, & n>M B S_{j}^{\prime}\end{cases}
$$

By substituting $n=M B S_{j}^{\prime}$ and $n=M B S_{j}^{\prime}+1$ into (15), and taking maximum, we obtain (9). For the special case $T_{j}=T_{j}^{\prime}$, one can obtain (10).

\section{Numerical Results}

In this section, we provide examples to show the adyantages of the proposed GCRA traffic shaper. We compare the proposed GCRA traffic shaper with feedback signal against two GCRA traffic shaper designs without feedback signal: one with EDD scheduler and one with regular FIFO multiplexer. The regulator of all three shaper designs are similar. For the GCRA traffic shapers without feedback signal, no information about $A D T_{j}(k-1)$ is available. Therefore, their algorithm in the regulator is modified by replacing $A D T_{j}(k-1)$ in (1) and (2) with $E T_{j}(k-1)+1$. We then consider the worst case that the buffer in the regulator is always nonempty for each VC. The number of active VCs is set to be 10 . The output traffic descriptor of VC $j,\left(X_{j}, T_{j}, \tau_{j}\right)$, satisfies $X_{j}=T_{0}+j+1, T_{j}=T_{0}+3 j, \tau_{j}=49\left(T_{j}-X_{j}\right)$ in Table 1(a) and $X_{j}=T_{0}+2 j, T_{j}=T_{0}+3 j, \tau_{j}=49\left(T_{j}-X_{j}\right)$ in Table 1 (b), where $T_{0}$ ranges from 0 to 27 to yield different output traffic loads. Computer simulations confirmed that the output cell streams of the proposed GCRA traffic shaper do not contain any non-conforming cells. However, the output cell streams of the two traffic shapers without feedback signal do contain non-conforming cells due to emission conflicts in the 
(a) $X_{j}=T_{0}+j+1, T_{j}=T_{0}+3 j, \tau_{j}=49\left(T_{j}-X_{j}\right), j=1, \cdots, 10$

\begin{tabular}{|c|c|c|c|c|c|c|c|c|c|}
\hline \multirow{2}{*}{$\begin{array}{c}\text { GCRA } \\
\text { Traffic Shaper }\end{array}$} & \multirow{2}{*}{$\begin{array}{c}\text { Simulation } \\
\text { Results }\end{array}$} & \multicolumn{8}{|c|}{$T_{0}$} \\
\hline & & 0 & 1 & 2 & 4 & 6 & 9 & 15 & 27 \\
\hline EDD With & Non-conforming cell ratio & 0 & 0 & 0 & 0 & 0 & 0 & 0 & 0 \\
\hline Feedback & Maximum queue size & 10 & 10 & 10 & 10 & 10 & 10 & 10 & 10 \\
\hline EDD Without & Non-conforming cell ratio & $1.0 \mathrm{e}-3$ & $8.6 e-5$ & $1.4 \mathrm{e}-4$ & $2.8 e-4$ & $3.1 \mathrm{e}-4$ & $3.1 e-4$ & $3.0 \mathrm{e}-4$ & $2.7 \mathrm{e}-4$ \\
\hline Feedback & Maximum queue size & 275 & 180 & 113 & 20 & 10 & 10 & 10 & 10 \\
\hline FIFO Without & Non-conforming cell ratio & $5.6 \mathrm{e}-3$ & $1.5 \mathrm{e}-2$ & $1.4 \mathrm{e}-2$ & $5.8 \mathrm{e}-3$ & $1.1 \mathrm{e}-3$ & $3.4 \mathrm{e}-4$ & $3.5 \mathrm{e}-4$ & $2.7 \mathrm{e}-4$ \\
\hline Feedback & Maximum queue size & 275 & 180 & 113 & 20 & 10 & 10 & 10 & 10 \\
\hline
\end{tabular}

(b) $X_{j}=T_{0}+2 j, T_{j}=T_{0}+3 j, \tau_{j}=49\left(T_{j}-X_{j}\right), j=1, \cdots, 10$

\begin{tabular}{|c|c|c|c|c|c|c|c|c|c|}
\hline \multirow{2}{*}{$\begin{array}{c}\text { GCRA } \\
\text { Traffic Shaper }\end{array}$} & \multirow{2}{*}{$\begin{array}{c}\text { Simulation } \\
\text { Results }\end{array}$} & \multicolumn{8}{|c|}{$T_{0}$} \\
\hline & & 0 & 1 & 2 & 4 & 6 & 9 & 15 & 27 \\
\hline \multirow{2}{*}{$\begin{array}{l}\text { EDD With } \\
\text { Feedback }\end{array}$} & Non-conforming cell ratio & 0 & 0 & 0 & 0 & 0 & 0 & 0 & 0 \\
\hline & Maximum queue size & 10 & 10 & 10 & 10 & 10 & 10 & 10 & 10 \\
\hline \multirow{2}{*}{$\begin{array}{c}\text { EDD Without } \\
\text { Feedback }\end{array}$} & Non-conforming cell ratio & $1.0 \mathrm{e}-2$ & $1.4 \mathrm{e}-3$ & $4.1 \mathrm{e}-4$ & $2.9 e-4$ & $2.9 \mathrm{e}-4$ & $2.7 \mathrm{e}-4$ & $2.6 \mathrm{e}-4$ & $3.1 \mathrm{e}-4$ \\
\hline & Maximum queue size & 150 & 50 & 11 & 10 & 10 & 10 & 10 & 10 \\
\hline \multirow{2}{*}{$\begin{array}{c}\text { FIFO Without } \\
\text { Feedback }\end{array}$} & Non-conforming cell ratio & $2.4 \mathrm{e}-2$ & $1.7 \mathrm{e}-2$ & $1.5 \mathrm{e}-2$ & $6.2 \mathrm{e}-3$ & $1.9 \mathrm{e}-3$ & $3.4 \mathrm{e}-4$ & $3.8 \mathrm{e}-4$ & $3.3 \mathrm{e}-4$ \\
\hline & Maximum queue size & 150 & 50 & 11 & 10 & 10 & 10 & 10 & 10 \\
\hline
\end{tabular}

Table 1: Comparison of the GCRA traffic shapers with and without feedback signals.

scheduler. The maximum queue size in the scheduler is controlled to be equal to 10 with feedback signal. While for the other traffic shapers without feedback signal, the maximum queue size can be much larger than the number of active VCs and the hardware design of the scheduler can be much more difficult.

\section{Conclusions}

In this paper, a new GCRA traffic shaper has been described and analyzed for supporting VBR traffic. This shaper has considered and resolved the emission conflict problem that other designs [1]-[5] did not. By using a feedback signal between the regulator and the scheduler, not only it can guarantee the output cell streams to conform to the output traffic descriptor, necessary call admission control can also be easily applied. Under the call admission control policy, the cell delay bound for each VC in the traffic shaper, including both the regulator and the scheduler, has been derived. One can observe that the cell delay bound of a specific VC in the traffic shaper is independent of the other VCs. This feature should be another advantage of the proposed design.

\section{References}

[1] F. Guillemin and W. Monin, " Management of Cell Delay Variation in ATM Networks, "IEEE GLOBECOM'92, pp. 128-132.

[2] P. E. Boyer, F. M. Guillemin, M. J. Servel, and J. Coudreuse, "Spacing Cells Protects and Enhances U- tilization of ATM Network Links, " IEEE Network, pp. 38-49. Sept. 1992.

[3] F. M. Brochin, "A Cell Spacing Device for Congestion Control in ATM Networks, "Performance Evaluation, pp. 107-127, 1992.

[4] G. Rigolio, L. Verri, and L. Fratta, "Source Control and Shaping in ATM Networks, "IEEE GLOBECOM'91, pp. 276-280.

[5] F. Bernabei, L. Gratta, M. Listanti, and M. Testa, “ Analysis of Two Level Shaping for Multiplexing of ONOFF ATM Sources, " IEEE ICC'93, pp. 1380-1385.

[6] G. Mercankosk, T. Moors, and A. Gantoni, "Multiplexing Spacer Outputs on Cell Emissions, "IEEE INFOCOM'95, pp. 49-55.

[7] The ATM Forum, ATM User-Network Interface Specification, Version 3.0, PTR Prentice-Hall, 1993.

[8] H. Saito, "Optimal Queuing Discipline for Real-Time Traffic at ATM Switching Nodes, " IEEE Trans. Commun., Vol. 38, No. 12, pp. 2131-2136, Dec. 1990. 